\title{
Analysis of Manufacturing Process of Shaft Parts Based on Thermal Spraying
}

\author{
Wang Guodong, Jin Fengfeng \\ Jiyuan Vocational and Technical college, Jiyuan459000, China
}

Keywords: remanufacturing technology; axial parts; service life; thermal spraying; finite element

\begin{abstract}
Thermal spray technology is one of the key supporting technologies of remanufacturing engineering, and it is an important technical means to realize the rapid recovery of the surface dimensions of the damaged parts, to improve the performance and prolong the service life. In this paper, the main shaft of the waste machine tool is used as an example, the outer surface of the shaft is repaired by plasma spraying technology. The finite element software ANSYS for thermal spray coating system of temperature and residual stress of linear elastic analysis, get the same profile nodes in different depths of temperature versus time curve, profile and different radial stress distribution curve for thermal spray remanufacturing process optimization provides more accurate reference data.
\end{abstract}

\section{Introduction}

Machine tool remanufacturing is mainly for the main parts of the machine tools to repair and re processing, through the repair can make parts to restore function, and even improve the performance of parts. Machinery parts and components from raw materials through heat treatment and machining of complex completed and entered the first service, due to wear and fatigue damage and other factors, the size and shape of parts performance will change, eventually leading to be eliminated. With the continuous development of the machine tool remanufacturing technology and thermal spraying technology, the machining process of the old parts of the machine tool can greatly improve the performance of the parts and prolong the service life of the parts.

The preparation of thermal spraying layer is mainly divided into six steps, including the processing of shaft surface pretreatment, surface rough treatment, spraying preparation work, coating preparation, coating of postprocessing and coating machine processing,

(1) Axis surface processing pretreatment

In order to coating and axis of the organic combination, improve the strength of the binding, the preprocessing treatment on the shaft surface spray, removal of shaft surface stains, the main method is using solvent cleaning cleaning, cleaning is required after the surface further processing.

(2) Surface rough treatment

The purified shaft need for rough processing, the surface of the shaft to form uniform convex surface, coating and shaft more closely combined, most commonly used rough processing includes sandblasting, electricity and galling, rough surface treatment on the shaft preheating.

(3) Spray preparation

Before the spraying, the axis is heated to 100 DEG C, shaft surface moisture removal, the shaft in the active state, before the spray coating, also need to work not to be sprayed with the local protection and material protection can be iron and brass sheet protective cover.

(4) Coating preparation

Choose the appropriate spraying materials, the shaft is fitted to the fixture, the use of appropriate heat source, spraying process parameters on the surface of the spray, spraying process as shown in figure 1. 


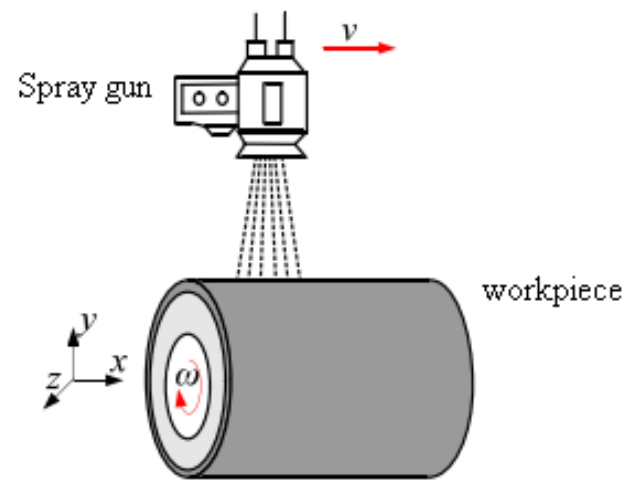

Fig. 1 Schematic diagram of spraying process

Figure 1 shows of the spraying process diagram in spraying need to choose a proper path for spraying, spraying quantity, workpiece speed and path can use NC machine tool programming design, so as to improve the manufacturing precision spraying.

(5) Coated postprocessing

Thermal spraying will produce a manufacturing error, in order to prevent spraying porous materials so that contact the shaft material corrosion and need to will be to produce the coating pore closed, sealing, we need to spray immediately after, commonly used hole sealing agent including paraffin, oil and phenolic resin.

(6) Coating machine processing

Coating spraying is completed, through mechanical processing to further improve the manufacturing precision, machining allowance is large, can use after the first turning grinding processing method, if the processing of a smaller margin, direct grinding working step, the selection of cutting tool and grinding wheel material need according to the coating material hardness of the workpiece and the size and shape.

\section{Mechanical analysis of thermal spraying shaft parts}

Because the shaft is a rotating body, so parts of the structure can be simplified as the shape of a hollow cylinder. When the model is completely after spraying, assuming the coating in the stress free state, when the coating cools, substrate and the layer will produce inconsistent strain, resulting in axial force, and the force model as shown in Figure 2.

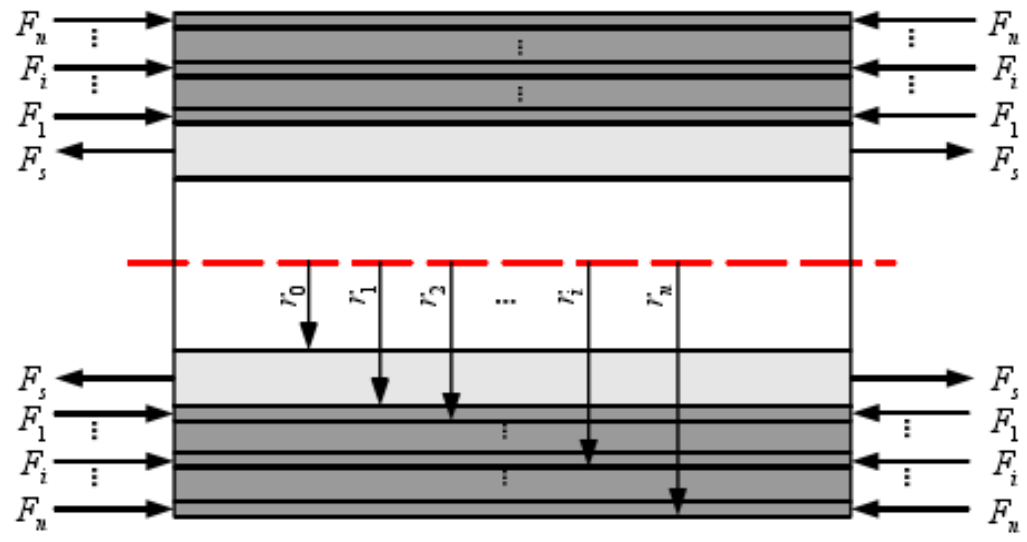

Fig. 2 Force analysis model of cylindrical coating

Figure 2 shows the force analysis model of the cylindrical coating, when the cylinder is not affected by the external force, according to the mechanical equilibrium of the system,we can get the formula (1). 


$$
F_{s}+\sum_{i=1}^{n} F_{i}=0
$$

In the formula (1), $\mathrm{s}$ is the basic body, $\mathrm{i}$ is coating, when determining the stress of the coating, the stress of the coating can also be obtained, and its expression is

$$
\left\{\begin{array}{c}
\left(\sigma_{s}\right)_{s}=\frac{F_{s}}{\pi\left(r_{1}^{2}-r_{0}^{2}\right)} \\
\left(\sigma_{s}\right)_{i}=\frac{F_{i}}{\pi\left({r_{i+1}}^{2}-r_{i}^{2}\right)} \quad(1 \leq i \leq n)
\end{array}\right.
$$

Each layer in the peripheral direction and radial direction of unconstrained independent deformation, it will produce a different strain, assuming the substrate surface is not pressure, outer surface by pressure according to the lame equation can be obtained radial and axial stress is hypothesis

$$
\left\{\begin{array}{c}
\left(\sigma_{r}\right)_{s}=\frac{p_{1} r_{1}^{2}}{r_{1}^{2}-r_{0}^{2}}\left(1-\frac{r_{0}^{2}}{r^{2}}\right) \\
\left(\sigma_{\theta}\right)_{s}=\frac{F_{i}}{\pi\left(r_{i+1}{ }^{2}-r_{i}^{2}\right)}\left(1+\frac{r_{0}^{2}}{r^{2}}\right)
\end{array}\right.
$$

The stress of the layer i of the coating is shown in Figure 3.

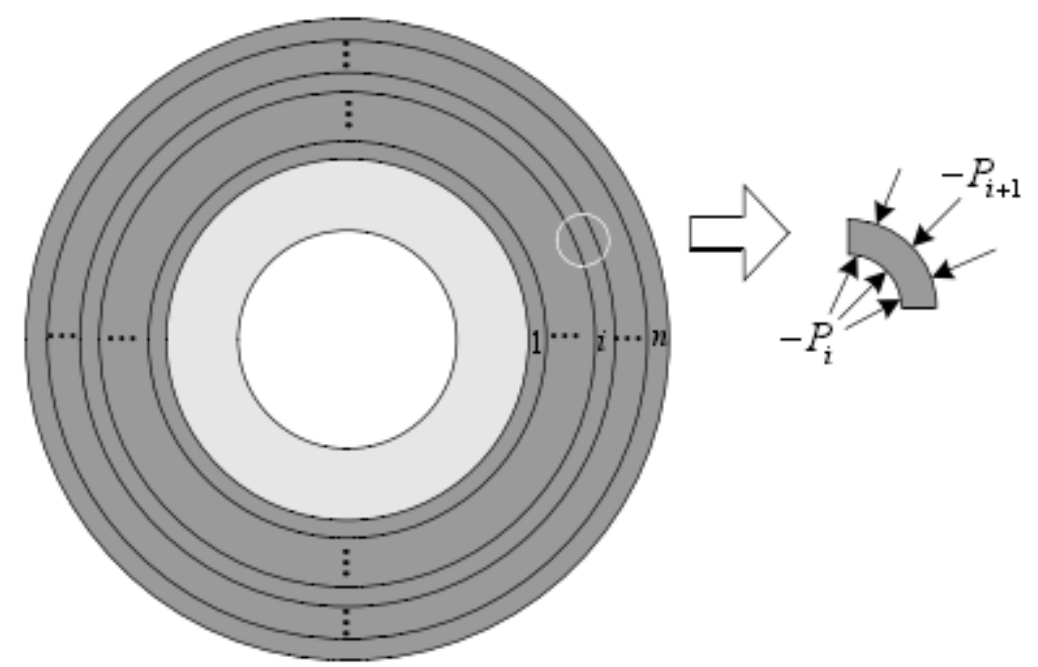

Fig.3 Schematic diagram of the stress of the layer I coating

It is assumed that the temperature variation range is $\Delta \mathrm{T}$, the strain of the substrate and the coating is mainly elastic stress and the thermal strain induced by temperature.

$$
\begin{aligned}
& \left\{\begin{array}{l}
\varepsilon_{s}=\frac{1}{E}\left[\sigma_{s}-v\left(\sigma_{\theta}+\sigma_{r}\right)\right]+\alpha \Delta T \\
\varepsilon_{\theta}=\frac{1}{E}\left[\sigma_{\theta}-v\left(\sigma_{s}+\sigma_{r}\right)\right]+\alpha \Delta T
\end{array}\right. \\
& \left\{\begin{array}{l}
\left(\varepsilon_{s}\right)_{i}=\frac{1}{E_{i}}\left[\left(\sigma_{s}\right)_{i}-v_{i}\left[\left(\sigma_{\theta}\right)_{i}+\left(\sigma_{r}\right)_{i}\right]\right]+\alpha_{i} \Delta T \\
\left(\varepsilon_{\theta}\right)_{i}=\frac{1}{E_{i}}\left[\left(\sigma_{\theta}\right)_{i}-v_{i}\left[\left(\sigma_{s}\right)_{i}+\left(\sigma_{r}\right)_{i}\right]\right]+\alpha_{i} \Delta T
\end{array}\right.
\end{aligned}
$$

In the formula ,E is elastic modulus, $\alpha$ is the coefficient of thermal expansion, and $v$ is the Poisson's ratio, due to the strain at the interface is continuous, so you can get the substrate and the first layer and 
the $i$ and the $i+1$ coating strain continuity equation and the expressions are shown in the following formula.

$$
\begin{aligned}
& \left\{\begin{array}{c}
\left(\varepsilon_{s}\right)_{s}=\left(\varepsilon_{s}\right)_{1} \\
\left(\varepsilon_{\theta}\right)_{s}=\left(\varepsilon_{\theta}\right)_{1}
\end{array}\right. \\
& \left\{\begin{array}{c}
\left.\left(\varepsilon_{s}\right)_{i}=\left(\varepsilon_{s}\right)_{i+1}\right) \\
\left(\varepsilon_{\theta}\right)_{i}=\left(\varepsilon_{\theta}\right)_{i+1}
\end{array}\right.
\end{aligned}
$$

According to the stress and strain of the shaft, the process can be optimized according to the design standard of the process, which can improve the machining quality.

\section{Thermal spraying shaft parts processed process temperature and stress analysis}

In order to analysis of thermal spraying shaft spray remanufacturing temperature and stress distribution, using ANSYS software on the part of the spraying process of virtual simulation, as a result of spraying coating amount of small thickness, so the model only considers coating and a portion of the shaft is combined with the stress distribution, away from the shaft can not be considered.
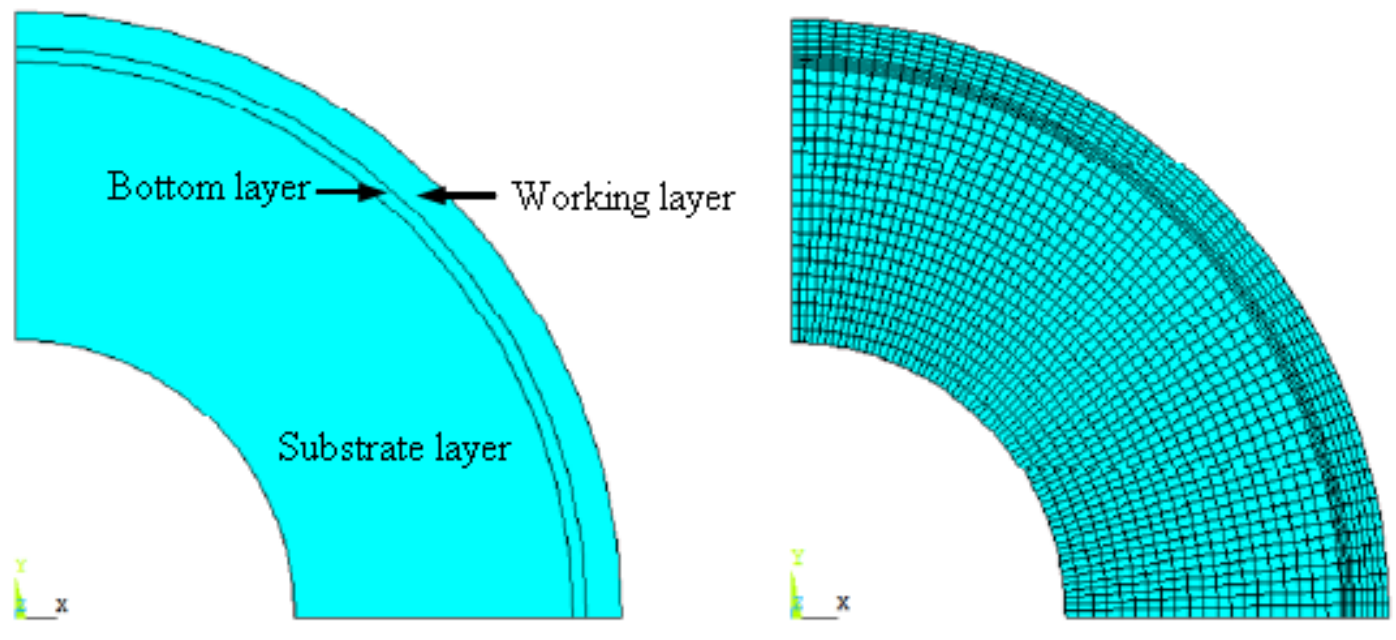

Fig. 4 Axial and coated ANSYS model

Figure 4 shows rachis and coating using ANSYS to establish the model in which the grid using tetrahedral unstructured mesh. The model includes body axis, the bottom layer and the working layer, coating and shaft using contact grid connection, in order to speed up the calculation of four points, the quarter symmetric strain model.

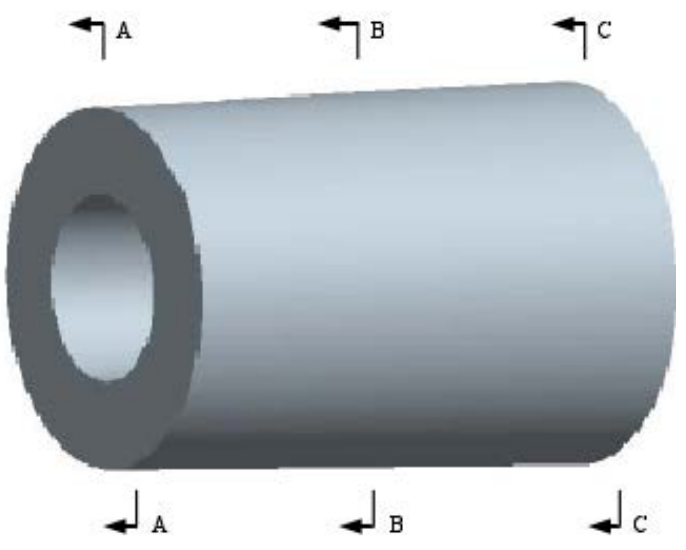

Fig.5 Schematic diagram of axis and coating profile model 
Figure 5 shows axis and the coating profile model diagram to display residual stress results more accurate, along the axis of shaft and coating took three slope, the number respectively A-A, B-B and C$\mathrm{C}$, first of all the temperature changes of the B-B profile nodes simulation, are obtained through calculation, such as shown in Figure 6.

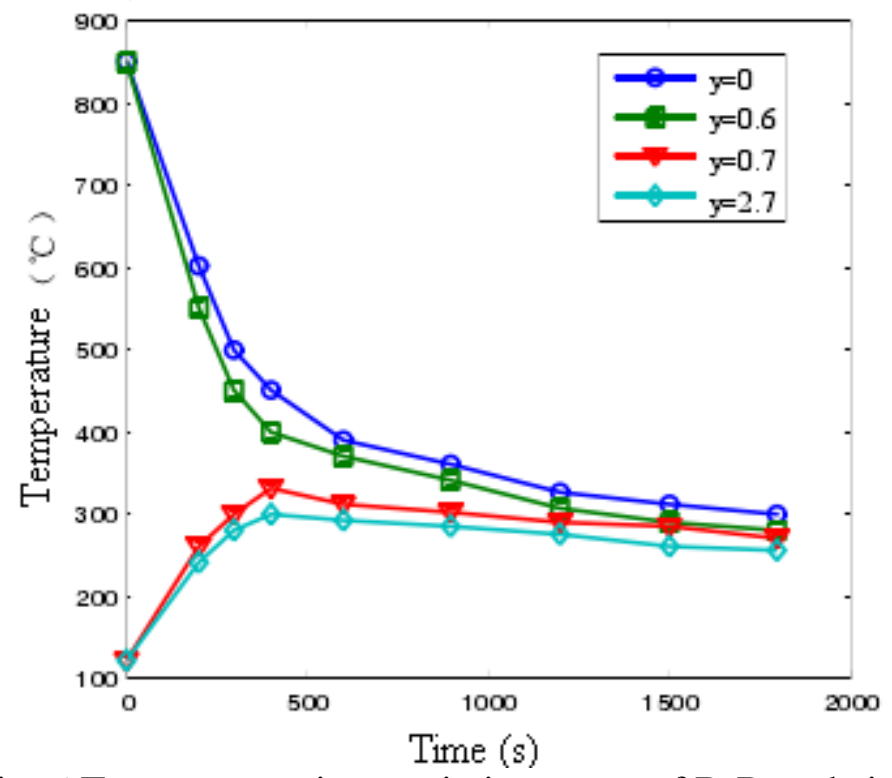

Fig. 6 Temperature time variation curve of B-B node in profile

Figure 6 shows in B-B distance on the surface of the coating at different depths and temperature change curve and its distance from the node with the surface are set to $0,0.6,0.7$ and $2.7 \mathrm{~mm}$, from figure can be seen coating in the 300s cooling fast, 300s cooling rate began to slow down. In the $1800 \mathrm{~s}$ to room temperature, cooling process end.

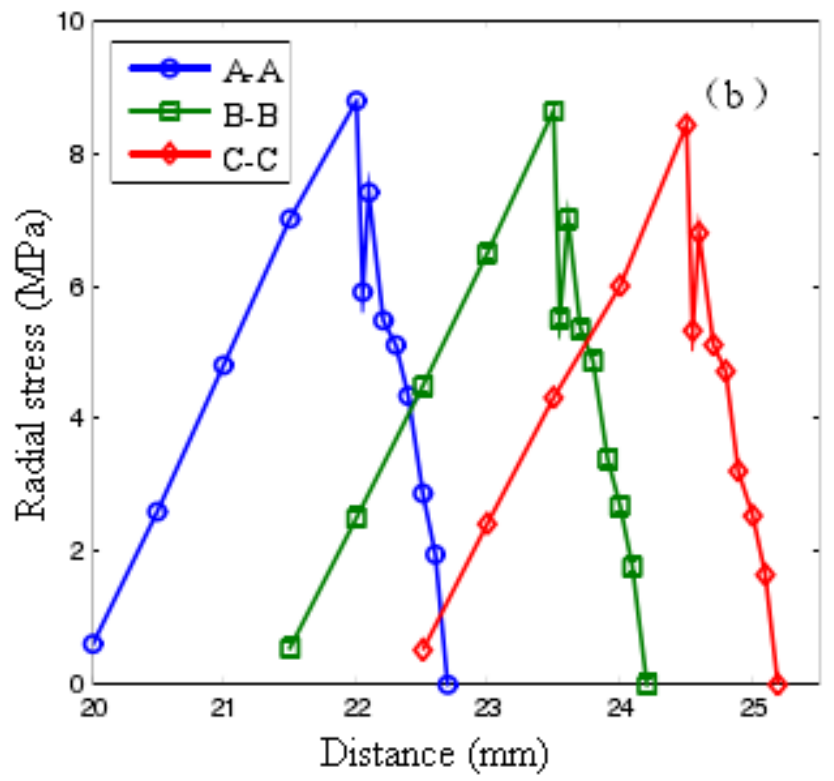

Fig 7 Schematic diagram of the radial stress of the shaft and coating

As shown in Figure 7, the coating system of radial stress is tensile stress, from inner shaft surface outside gradually increased, in backing layer reaches a maximum value, and then decreased gradually. Finally reduced to 0 . According to the cooling rate of the workpiece and the radial stress of the spraying and spraying, the machining process can be reasonably arranged, which provides the necessary reference for the optimization of the process flow. 


\section{Conclusions}

By plasma spraying technique to repair severe wear of machine tool spindle, and the establishment of the coating of the force model. Finally, the finite element ANSYS software of spraying process of temperature and stress distribution of the simulation. Finite element method simulation results show that the cooling rate of the coating in the 300s after the beginning of the slow, at 1800s to room temperature, the end of the cooling process. In the coating system, the radial stress is tensile stress, which is gradually increased from the inner surface of the shaft, and the maximum value is located at the junction of the substrate and the coating.

\section{Reference}

[1] Cao Huajun, Du Yanbin, Zhang Mingzhi, et al. The connotation and technical framework of machine tool remanufacturing and synthesis [J]. China Surface Engineering, 2010, 23 (6): 75-79.

[2] in Hong Jian, Jia Junhong, Zhang Zhenyu, et al. Research progress of thermal spraying technology and thinking of [J]. materials review, 2013, 27 (2): 38-40.

[3] Niu Liping, Zhang Tingan, Shi Guanyong, et al. Residual stress in plasma sprayed coatings for thin wall parts [J]. Journal of Northeastern University (NATURAL SCIENCE EDITION), 2011,32 (10): 1448-1451.

[4] Dong Wen. Application of arc spraying technology in the remanufacturing of machine tool spindle [J]. machinery manufacturing, 2009, 47 (8): 70-72.

[5] Wan Chunfeng, Xin Lei. Several new generation of thermal spraying technology J. Mechanical engineering and automation, 2012 (3): 206 - 208

[6] Bao Junfeng, Cui Ying, Hou Yubai, et al. Development and present situation of supersonic thermal spraying technology (J). Thermal spraying technology, 2011, 3 (4): 21 - 18

[7] Zhang Xianglin, Li Wei, Zhao Jie, et al. Application of the supersonic flame spraying technology in the mold repair (J). Die \& mold industry, 2010, 36 (9): 62 - 65

[8] Liang Xiubing, Chen Yongxiong, Bai Jinyuan, et al. Automatic high speed arc spraying technology for re manufacture of engine crankshaft (J). China Surface Engineering, 2010, 23 (2): 116 - 112

[9] Miao Lou, Al, Qiang Wen Jiang, et. High velocity arc spray coating in the steel structures against corrosion effect and its application [J]. Protective material, 2011, 44 (3): 54 - 46.

[10] Han Xiaoling, Liu Xia. Method of repairing machine hydrostatic spindle thermal spraying [J]. manufacturing technology and machine tool, based on the 2013 (2): 98-100.

[11] Wu Huiyuan. Study on the modification of the multiple composite coating on the spindle of NC machine tools [J]. hot working technology, 2013,42 (16): 165-167.

[12] Sun Yin. Thermal spray repair of worn shaft neck of heavy duty transmission parts [J]. material protection, 2008,41 (12): 73-74. 